\title{
Viability of Toxoplasma gondii tachyzoites in different conditions for parasite transportation
}

\author{
Thi Thuy Nguyen ${ }^{1,2}$ (D), Ketsarin Kamyingkird ${ }^{1}$ (D), Waraphon Phimpraphai ${ }^{(i D}$ and Tawin Inpankaew ${ }^{1}$ (D)
}

1. Department of Parasitology, Faculty of Veterinary Medicine, Kasetsart University, Bangkok 10900, Thailand; 2. Department of Veterinary Medicine, Faculty of Animal Science and Veterinary Medicine, University of Agriculture and Forestry, Hue University, Hue, Vietnam; 3. Department of Veterinary Public Health, Faculty of Veterinary Medicine, Kasetsart University, Bangkok 10900, Thailand.

Corresponding author: Tawin Inpankaew, e-mail: tawin.i@ku.th

Co-authors: NTT: ntthuy.huaf@hueuni.edu.vn, KK: ketsarinkamy@hotmail.com, WP: fvetwrp@ku.ac.th Received: 28-08-2021, Accepted: 31-12-2021, Published online: 29-01-2022

doi: www.doi.org/10.14202/vetworld.2022.198-204 How to cite this article: Nguyen TT, Kamyingkird K, Phimpraphai W, Inpankaew T (2022) Viability of Toxoplasma gondii tachyzoites in different conditions for parasite transportation, Veterinary World, 15(1): 198-204.

\begin{abstract}
Background and Aim: Toxoplasma gondii tachyzoite is the infective stage that causes acute infection, leading to severe toxoplasmosis. The tachyzoite stage has been extensively used for several inoculation purposes, including antigen production, immunological studies, nutrition mechanisms, and in vitro drug trials. The use of fresh tachyzoites is required for inoculation in either in vitro or in vivo studies. However, there is a lack of information on preserving live tachyzoites during transportation from laboratories to inoculation sites. Therefore, this study aimed to validate suitable preservative conditions for maintaining live parasites by determining the survival and viability of $T$. gondii tachyzoites on the basis of different media, temperatures, and incubation times.
\end{abstract}

Materials and Methods: The free live T. gondii tachyzoites were evaluated on their viability when maintained in different media without 5\% Carbon dioxide $\left(\mathrm{CO}_{2}\right)$. The purified tachyzoites of the RH and PLK strains were individually suspended in normal saline (NS), phosphate-buffered saline (PBS), minimum essential medium (MEM), and MEM with $10 \%$ fetal bovine serum (MEM-FBS) and incubated for $6 \mathrm{~h}$ at ice-cold $\left(\mathrm{IC} ; 3-9^{\circ} \mathrm{C}\right)$ and room temperature $\left(\mathrm{RT} ; 25^{\circ} \mathrm{C}\right)$. Parasite survival was measured at the $0,1^{\text {st }}, 2^{\text {nd }}, 3^{\text {rd }}, 4^{\text {th }}, 5^{\text {th }}$, and $6^{\text {th }} \mathrm{h}$ post-incubation using the trypan blue exclusion test.

Results: The viability was in the range of $85.0 \%-91.0 \%$ for IC using NS and $81.0 \%-85.1 \%$ (IC) and $75.3 \%-77.5 \%$ (RT) using PBS. The viability was approximately $75.0 \%-83.0 \%$ (IC) and $70.0 \%-79.0 \%$ (RT) using MEM and MEM-FBS. There was a significant difference in the viability between the seven periods on the basis of one-way repeated Analysis of variance and Friedman analyses. Parasite survival slightly reduced (20.0\%-30.0\%) in NS and MEM-FBS at both temperatures during incubation. Notably, PBS could not support tachyzoite viability after $3 \mathrm{~h}$ post-incubation.

Conclusion: NS was a suitable preservative for maintaining purified $T$. gondii tachyzoites during transportation at IC and RT without $5 \% \mathrm{CO}_{2}$ supplementation. This could be a valuable medium for parasite transportation, especially when there is a large distance between the laboratory and inoculation site.

Keywords: preservation time, T. gondii tachyzoites, temperature, transportation, viability.

\section{Introduction}

Toxoplasma gondii, an obligate intracellular protozoan, is a ubiquitous parasite that infects almost all warm-blooded animals, including humans. Most of the infected immunocompetent individuals develop either asymptomatic or mild clinical signs, whereas acute infection can cause encephalitis and retinochoroiditis, particularly in immunocompromised hosts, and congenital disease in seronegative pregnant women $[1,2]$. Furthermore, the proliferation and development of the tachyzoite stage induce a reduction in liver and brain cholesterol content and a decline in host immune responses, leading to acute infection [3].

Copyright: Nguyen, et al. Open Access. This article is distributed under the terms of the Creative Commons Attribution 4.0 International License (http://creativecommons.org/licenses/ by/4.0/), which permits unrestricted use, distribution, and reproduction in any medium, provided you give appropriate credit to the original author(s) and the source, provide a link to the Creative Commons license, and indicate if changes were made. The Creative Commons Public Domain Dedication waiver (http:// creativecommons.org/publicdomain/zero/1.0/) applies to the data made available in this article, unless otherwise stated.
During the T. gondii life cycle, three asexual stages can invade the cells, including sporozoite, which is only produced by sexual reproduction and released in the oocysts through felid feces; bradyzoite, a form of slow multiplication originating from the tissue cysts in chronic infection; and tachyzoite, a form of proliferation that is characteristic of the acute infection $[4,5]$. The tachyzoites can be maintained and produced in cell cultivation systems (in vitro) and animal models (in vivo), which makes it the most experimentally tractable organism among Apicomplexa and has been modeled in many studies [4]. Tachyzoites can be purified and used in several studies, including immunity responses, histopathology, and drug trials. Suspension of the purified tachyzoites in the appropriate media helps avoid unexpected responses of animal or host cells to the parasites [6-8]. To maintain high viability and survival rates for the parasites, $T$. gondii tachyzoites should be used for inoculation in an animal model $[9,10]$ and in vitro study immediately after purification. 
Heider et al. [9] and Räisänen [11] demonstrated that tachyzoites survive and remain infectious better in serum solutions than the free nutrient media, the viability of the parasites in phosphate-buffered saline (PBS) or normal saline (NS) was significantly high, compared to enriched media in other studies $[12,13]$. Besides, the sensitivity of $T$. gondii tachyzoites against low $\mathrm{pH}$ was found in an experiment in which simulated gastric fluid was used to examine the tachyzoites infectivity retention in different acidity [14]. In general, these studies revealed the sensitivity of purified tachyzoites to the extracellular environment; to keep them alive outside the host cells; the tachyzoites must be maintained in the cultivation media in a sealed container without Carbon dioxide $\left(\mathrm{CO}_{2}\right)$ supplementation during transportation. Some experiments require long-distance and time-consuming transportation to carry the purified, live parasites to the experimental animal areas from the laboratory. Unfortunately, a standardized protocol or set of conditions for maintaining live, purified tachyzoites for long-distance transportation is unavailable. Therefore, appropriate media and temperatures to maintain the $T$. gondii purified tachyzoites' viability and infectivity during long-distance transportation need to be identified.

The objective of this study was to validate the conditions for preserving live, purified $T$. gondii tachyzoites in different media at two different temperatures and incubation periods without a $5 \% \mathrm{CO}_{2}$ supplement.

\section{Materials and Methods}

\section{Ethical approval}

This study was carried out under a project on toxoplasmosis in duck that adhered to strict guidelines of animal care and use under the Ethical Review Board of the Office of National Research Council of Thailand (NRCT) for the conduction of the scientific research. The Approval number of ACKU63-VET035 was granted by Kasetsart University's Institution Animal Care and Use Committee. Besides, the biosafety number IBC-63-V04 was granted by Institutional Biosafety Committee, Faculty of Veterinary Medicine, Kasetsart University.

\section{Study period and location}

The study was conducted from March to May 2020 . The viability of $T$. gondii tachyzoites maintained in different conditions was measured in the laboratory at Department of Parasitology, Faculty of Veterinary Medicine, Kasetsart University.

\section{Preservative preparation}

Four different media were used to preserve the T. gondii tachyzoites: PBS $1 \mathrm{X}(\mathrm{pH} 7.4)$, NS $0.85 \%$, with adjusted $\mathrm{pH}$ 7.4, minimum essential medium (MEM 1X with Earle's salts and 2.0-mM L-glutamine [Gibco ${ }^{\mathrm{TM}}$, USA]), and MEM-fetal bovine serum (FBS) (MEM+10\% FBS [Gibco]). PBS and NS were autoclaved at $121^{\circ} \mathrm{C}$ for $15 \mathrm{~min}$ at 1.1 bars using a
Tomy SX-500 high-pressure steam sterilizer (U.P. Marketing General Supply Co. Ltd., Thailand). MEM and MEM-FBS were passed through $0.2-\mu \mathrm{m}$ syringe filters (Millipore, United States). As MEM-FBS contains bovine serum albumin (BSA), it is not tailored for in vivo assay but for other biological experiments of $T$. gondii, so this medium was also investigated to compare with the other buffers. Freshly prepared preservatives were used in every experiment to prevent $\mathrm{pH}$ changes.

\section{T. gondii strains and parasite preparation}

Tachyzoites of the T. gondii RH and PLK strains were used in this study. The parasites were maintained using vero cells in MEM cultivation media supplemented with $1 \%$ Pen Strep (Gibco), 0.1\% anti-fungi $\left(250-\mu \mathrm{g} / \mathrm{mL}\right.$, HyClone ${ }^{\mathrm{TM}}$, USA), and 5-8\% FBS. Both T. gondii strains and vero cells were obtained from the Department of Protozoology, Faculty of Tropical Medicine, Mahidol University, Bangkok, Thailand. The $T$. gondii tachyzoites were cultivated every 4 days in vero cells. Harvesting of the tachyzoites was performed as described by Nguyen et al. [15]. The harvested tachyzoites were washed in cold media (PBS, NS, MEM, and MEM-FBS) using centrifugation 3 times at $448 \mathrm{~g}$ and $4^{\circ} \mathrm{C}$ for $10 \mathrm{~min}$. The number of parasites was counted using a Neubauer chamber (0.1mm depth, Blaubrand ${ }^{\circledR}$, Germany), then diluted to $3 \times 10^{6}$ tachyzoites $/ \mathrm{mL}\left(3 \times 10^{3}\right.$ tachyzoites $\left./ \mu \mathrm{L}\right)$ before transferring to the four different preservatives being tested. The parasites were preserved in $100 \mu \mathrm{L}$ of each medium and maintained in $1.5-\mathrm{mL}$ sterile tubes (Corning Life Science [Wujiang] Co., Ltd, China). The parasites were preserved at ice-cold (IC) conditions, where the temperature increased from $3^{\circ} \mathrm{C}$ in the $1^{\text {st }} \mathrm{h}$ to $9^{\circ} \mathrm{C}$ in the $6^{\text {th }} \mathrm{h}$ of incubation and room temperature (RT; $\left.25^{\circ} \mathrm{C}\right)$.

\section{Trypan blue exclusion test}

The viability of $T$. gondii tachyzoites was observed in triplicate using the trypan blue exclusion test as described by Strober [16]. An amount (100$\mu \mathrm{L}$ ) of $0.4 \%$ trypan blue (Gibco) was mixed well into each parasite suspension and incubated for $3 \mathrm{~min}$ at RT. The unstained and stained cells were considered live and dead tachyzoites, respectively. After staining, the parasites were counted within 3-5 min in a Neubauer chamber using the $40 \times$ objective lens of a light microscope (Olympus, Japan). Approximately 150 tachyzoites were counted in all subsquares of $1 \times 1 \mathrm{~mm}$ in the central square of the chamber. The viability was calculated as the ratio of live tachyzoites per total tachyzoites count and expressed as the mean viability. The viability of tachyzoites was monitored at the $0,1^{\text {st }}, 2^{\text {nd }}, 3^{\text {rd }}, 4^{\text {th }}, 5^{\text {th }}$, and $6^{\text {th }}$ hours post-incubation (hpi).

\section{Statistical analysis}

Statistical analyses were conducted using the $\mathrm{R}$ software package version 3.6.3 ( $\mathrm{R}$ Foundation for Statistical Computing, Vienna, Austria) [17]. 
Descriptive statistics, consisting of mean, median, standard error, the $25^{\text {th }}$ and $75^{\text {th }}$ quartiles, maximum and minimum of the viability of tachyzoites were reported. The normal distribution and homogeneity of variances were verified using the Shapiro test and Levene's test, respectively. The Kruskal-Wallis test followed by Wilcoxon's test was used to determine statistical significance between the viability of tachyzoites preserved in the four media. The $T$. gondii tachyzoite viability measured 7 times was analyzed using one-way repeated measures Analysis of variance (ANOVA) or the Friedman test, and $* p<0.05, * * p<0.01$, and $* * * p<0.001$ were tested to indicate significant differences.

\section{Results}

Effect of different media on the viability of $T$. gondii tachyzoites preserved at IC and RTs

The comparison of $T$. gondii tachyzoite viability in the different preservatives under the IC and RT conditions is shown in Figure-1 using the mean viability of parasites at the seven measurement periods. At RT, NS had the highest viability at $84.3 \%$ and $91.3 \%$ for the PLK and RH strains, respectively. The second highest viability in the PLK strain was $75.3 \%$ in PBS, yet this medium had the lowest proportion at $77.5 \%$ in the RH strain. In MEM and MEM-FBS, the viability of the PLK strain was about $70 \%$, which was slightly lower than that of the RH strain (approximately 79\%) (Figures-1a and $b$ ).

Similarly, at IC conditions, the highest viability was observed in NS with $85.8 \%$ and $90.6 \%$ for the PLK and RH strains, respectively, followed by viability in the PBS medium with $81.0 \%$ and $85.1 \%$ for the PLK and RH strains, respectively. For MEM-FBS, 76.4\% of PLK tachyzoites and $83.2 \%$ of RH tachyzoites were confirmed viable during incubation. The viability of tachyzoites in MEM was $75.5 \%$ and $80.5 \%$ for the PLK and RH strains, respectively, which were the lowest proportions at this temperature (Figures-1a and b).

There was a significant difference between the mean viability of tachyzoites preserved using the four types of media for both temperature conditions as assessed using the Kruskal-Wallis test. Further examination on the basis of the Wilcoxon test showed that the mean viability was significantly higher in the NS medium than in the MEM-FBS and MEM, notably for the RH strain at IC $(p<0.01)$ and RT $(p<0.001)$ (Figure-1b).

\section{Viability of $T$. gondii tachyzoites during the preser- vation period}

The viability of parasites in the different media was also monitored at different times. In general, the parasite's survival decreased from $90-100 \%$ to $50-85 \%$ over the $6 \mathrm{~h}$ of incubation.

There was no noticeable difference in the viability of parasites preserved using NS between the different temperatures. The viable rates of tachyzoites in this medium decreased steadily from just under $100 \%$ to over $70 \%$ (PLK strain) and $<80 \%$ (RH strain) at the $6^{\text {th }}$ hpi (Figures-2a and 3a). At IC condition, PBS was only able to support viability at $3 \mathrm{hpi}$, but with a mild decline from about $100 \%$ to approximately $88 \%$ (both T. gondii strains), which was followed by substantial drops to $51.5 \%$ (PLK strain) and $68.8 \%$ (RH strain) in the past $3 \mathrm{~h}$. At RT, the viability of tachyzoites in PBS medium fell moderately to $70 \%$ in both strains at the $3^{\text {rd }} \mathrm{hpi}$, then fluctuated and was lowest at $48.1 \%$ and $54.8 \%$ for PLK and RH strains, respectively, at the end of the period (Figures-2b and $3 b$ ).

With the MEM-FBS medium, there were progressive reductions from approximately $90 \%$ to $60 \%$ (PLK strain) and to $71.7 \%$ (RH strain) in the viable rates of parasites for the IC condition over the incubation period. The parasite viability levels maintained at RT dropped to $73.6 \%$ and $50.9 \%$ for PLK strain; and $85.2 \%$ and $71.7 \%$ for $\mathrm{RH}$ strain at the $3^{\text {rd }}$ and $6^{\text {th }}$ hpi, respectively (Figures-2c and 3c). Similarly, there were continuous reductions of approximately $30 \%$ and $20 \%$ in the viability of tachyzoites in MEM at IC, whereas for the RT conditions, there were gradual declines from more than $90 \%$ to $55.3 \%$ and $64.3 \%$ in the viable rates of PLK and RH strains, respectively (Figures-2d and 3d).

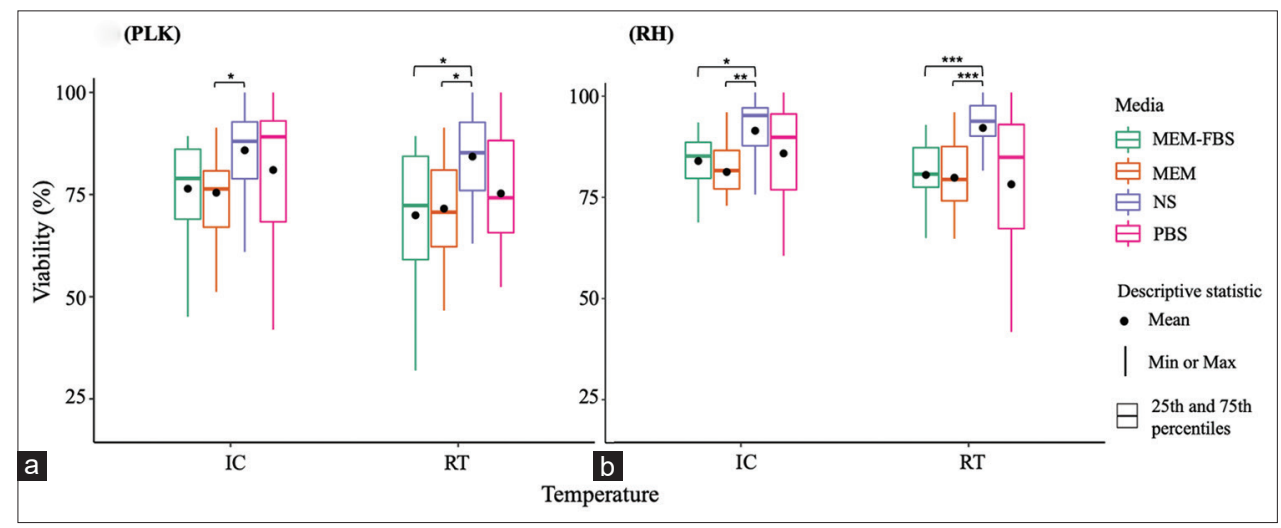

Figure-1: ( $a$ and b) Viability of Toxoplasma gondii tachyzoites preserved in four different media. Data of each medium at seven times are displayed as a box plot of the median (line), mean (dot), $25^{\text {th }}$ and $75^{\text {th }}$ percentiles (box), maximum and minimum (upper and whiskers, respectively). 


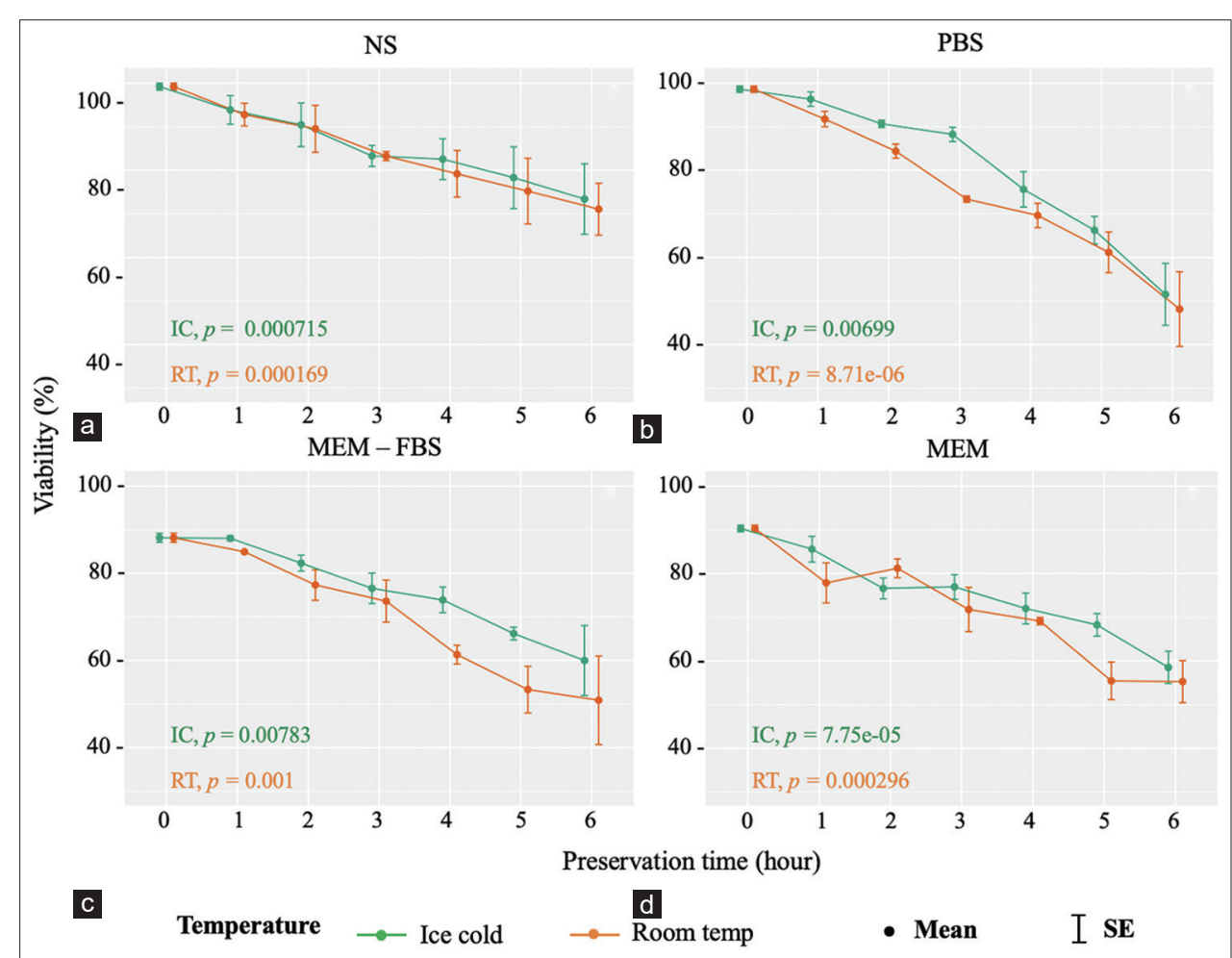

Figure-2: (a-d) Viability of Toxoplasma gondii PLK strain preserved in four different media ( $p$ indicates significant differences).

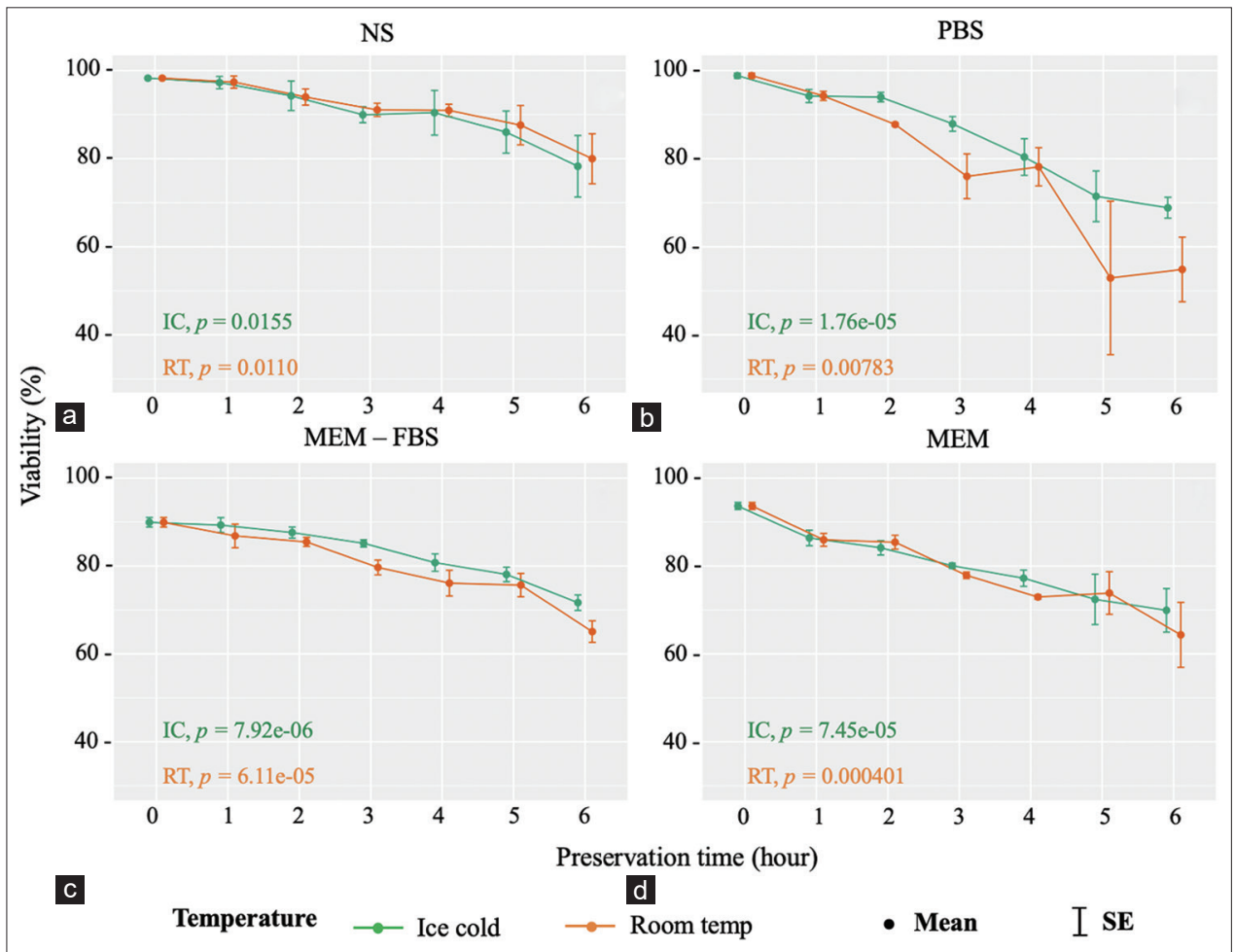

Figure-3: (a-d) Viability of Toxoplasma gondii RH strain preserved in four different media ( $p$ indicates significant differences).

One-way repeated ANOVA and the Friedman test showed that the levels of viable parasites were significantly different at different times $(\mathrm{p}<0.05$ as shown in every chart).

\section{Discussion}

Several factors, such as temperature, media, $\mathrm{pH}$, and time, may impact the survival of $T$. gondii tachyzoites $[9,13,18]$. The present result showed that NS was the best medium for short-term storage (within $6 \mathrm{~h}$ ) of $T$. gondii tachyzoites without $5 \% \mathrm{CO}_{2}$ supplementation. The viability of the parasites in this solution was significantly higher than in the other media made from MEM comprising many ingredients. NS is preferred as an isotonic solution, widely used in medical and pediatric practice, and in scientific research, 
especially for animal inoculation $[19,20]$. The results were comparable to other studies in which nutrient-free media, such as NS and PBS, were more suitable than minimum essential media for maintaining $T$. gondii tachyzoites. Indeed, the media without nutrients, vitamins, carbohydrates, and fats could prolong the viability of free $T$. gondii tachyzoites better than those containing these kinds of supplements $[12,13]$. The osmotic coefficient of NS is about 0.93 [21] and is similar to the osmolarity of blood in which circulating tachyzoites have been found in a range of host species [22-26]. However, NS is usually an acidic solution with a $\mathrm{pH}$ of about 5.5 that might be potentially harmful to the $T$. gondii tachyzoites [14,27]. Indeed, $T$. gondii tissue cysts have a high $\mathrm{pH}$ tolerance, but the tachyzoites were extremely sensitive to a solution with a pH of less than 6.0 [14,28]. Therefore, a fresh medium with an adjusted $\mathrm{pH} 7.4$ is vital for the short-term storage of tachyzoites because the $\mathrm{pH}$ easily falls when NS absorbs carbon dioxide in the atmosphere [27].

The second highest mean survival was found in the parasites incubated in PBS, except for the RH strain at RT. Interestingly, in contrast with the subtle variations in parasite survival in NS over the preservation period at both temperatures, PBS supported tachyzoite viability for only a short time, particularly at RT. Hence, the inoculation of tachyzoites maintained in PBS should be conducted within $3 \mathrm{~h}$ at IC or $1 \mathrm{~h}$ at RT after harvesting the parasites.

The $T$. gondii tachyzoites can invade any nucleated cells in the host or cell media and reside in the parasitophorous vacuole membrane (PVM). This membrane is host-derived yet extensively modified by parasites to propagate nutrient acquisition and avoid host immunity [29]. Tachyzoites replicate exclusively inside the PV and then lyse the host cell before the next round, increasing their number and extent of intracellular life [30,31]. PVM is freely permeable to vitamins, sugars, amino acids, nucleobases, nucleosides, and nucleotides that support parasite proliferation [32]. These small nutrients are transported passively from extracellular to inside the cell via the PVM pores constituted by parasite proteins, including GRA17 and GRA24 [33-35]. In addition, the uptake of proteins and lipids is involved in an intravacuolar membranous tubule-vesicular network consisting of membranous tubules and vesicles connecting the PVM and the parasites [36,37].

Contrastly, it was suggested that the resistance of extracellular tachyzoites is quite restricted in cellfree media and that the live tachyzoites could be undetected after $24 \mathrm{~h}$ of incubation; furthermore, they cannot proliferate without host cells $[10,13,38]$. However, free-living protozoans have demonstrated a higher number of computationally annotated transporter families in the genomes than the parasitic organisms [39], greatly stimulating the metabolism of these extracellular parasites. Clearly, the more nutrients they obtain from the media, the more metabolic materials they excrete. It was likely that these biological activities produce harmful materials and cause changes in solution $\mathrm{pH}$, threatening the parasite's survival. In addition, it showed that tachyzoites could survive longer at $4{ }^{\circ} \mathrm{C}$ than at $18-22^{\circ} \mathrm{C}$ and $37^{\circ} \mathrm{C}$ because they retain a minimum metabolic rate $[12,13]$. Therefore, enrichment of the nutrient solution, such as with the MEM or MEM-FBS media, and at RT might be more harmful to free-living tachyzoites than NS and PBS for the IC condition.

In addition, the MEM used in this experiment comprised both L-glutamine and Earle's salts, which are crucial for mammalian cell cultivation. However, they somehow might impact negatively on cells in the closed system. First, L-glutamine is an amino acid serving as an auxiliary energy source for protein and nucleic acid syntheses when the cells rapidly multiply. Nonetheless, L-glutamine is not stable in solution, unlike most other amino acids. Glutamine degradation results in the equimolar formation of ammonia, which is toxic to cells $[37,38]$. In fact, the rate at which degradation proceeds depends on time, temperature, type, and the $\mathrm{pH}$ of the buffer. Degradation of $0.23 \%$ /day occurred in water at $\mathrm{pH} 6.5$ and $0.8 \%$ /day in mixed total parenteral nutrition solution at $22-24^{\circ} \mathrm{C}$. The L-glutamine degradation rate in the intravenous solutions was $<0.15 \% /$ day at $4{ }^{\circ} \mathrm{C}[40,41]$. Therefore, the parasites incubated in MEM and MEM-FBS with IC conditions had higher survival proportions than at RT in this study.

Another important point is that Earle's salts have a high sodium bicarbonate level, so they should be used in a $5 \% \mathrm{CO}_{2}$ air environment. Because MEM supplemented with these salts was available for the cell cultivation system in the laboratory, it was compared with other solutions in this study. However, MEM with Hank's salts having a lower sodium bicarbonate level has been recommended in the closed system without $5 \% \mathrm{CO}_{2}$ to enhance the viability of tachyzoites [42].

The viability of tachyzoites in MEM-FBS was relatively higher than for MEM, which had the lowest proportion in almost all groups. The result agreed with other studies that reported that the addition of serum helped prolong free, living tachyzoites $[9,10,13]$. BSA, a principal component of FBS, is considered a microcarrier, making macromolecules more available for tachyzoites; thus, it could increase parasite survival [43].

\section{Conclusion}

NS was preferable to the other media for shortterm storage of free, purified T. gondii tachyzoites for IC and RT conditions. Using this preservative with the IC condition is likely to help prolong the viability of T. gondii tachyzoites by several hours without a $5 \%$ $\mathrm{CO}_{2}$ supplementation, which may benefit long-distance transportation. Because extracellular tachyzoites 
are extremely sensitive to the acidic media, the $\mathrm{pH}$ of the used media should be measured at the end of the incubation period to observe its change. This was a limitation in this study because of the small volume of residual media. Trypan blue exclusion is a simple and rapid technique to evaluate the viability of parasites. Yet, the viable tachyzoites are assessed subjectively, and small amounts of dye uptake indicating cell injury may go undetected. Thus, the infectivity of parasitic $T$. gondii tachyzoites might be needed to confirm with in vivo assay in animals.

\section{Authors' Contributions}

TI and KK: Planned and designed the study and revised the manuscript. WP: Analyzed the data. NTT: Conducted the study, analyzed the data, and drafted the manuscript. All authors have read and approved the final manuscript.

\section{Acknowledgments}

We would like to thank the Department of Protozoology, Faculty of Tropical Medicine, Mahidol University for providing the original Toxoplasma gondii strains and vero cells. This study was financially supported by the Faculty of Veterinary Medicine, Kasetsart University, Bangkok, Thailand, (grant number: TWP.63_06) and Ms. Thi Thuy Nguyen's scholarship to attend the master course in Veterinary Parasitology at Kasetsart University - "In-Country/ In Region Scholarships Programme at SEAMEO SEARCA, 2019 (grant number: 57454966)" offered by DAAD (German Academic Exchange Service) and SEARCA (Southeast Asian Regional Center for Graduate Study and Research in Agriculture).

\section{Competing Interests}

The authors declare that they have no competing interests.

\section{Publisher's Note}

Veterinary World remains neutral with regard to jurisdictional claims in published institutional affiliation.

\section{References}

1. Dubey, J.P. (2014) The history and life cycle of Toxoplasma gondii. In: Weiss, L.M. and Kim, K., editors. Toxoplasma gondii. $2^{\text {nd }}$ ed., Ch. 1. Academic Press, Boston. p1-17.

2. Laboudi, M., Taghy, Z., Duieb, O., Peyron, F. and Sadak, A. (2021) Toxoplasma gondii seroprevalence among pregnant women in Rabat, Morocco. Trop. Med. Health, 49(1): 21.

3. Milovanović, I., Busarčević, M., Trbovich, A., Ivović, V., Uzelac, A. and Djurković-Djaković, O. (2017) Evidence for host genetic regulation of altered lipid metabolism in experimental toxoplasmosis supported with gene data mining results. PLoS. One., 12(5): e 0176700.

4. Attias, M., Teixeira, D.E., Benchimol, M., Vommaro, R.C., Crepaldi, P.H. and de Souza, W. (2020) The life-cycle of Toxoplasma gondii reviewed using animations. Parasit. Vectors, 13(1): 588.

5. Martorelli, D.G.B. and Knoll, L.J. (2020) Comparisons of the sexual cycles for the Coccidian parasites Eimeria and Toxoplasma. Front. Cell. Infect. Microbiol., 10(776):
604897.

6. Benevides, L., Cardoso, C.R., Milanezi, C.M., Castro-Filice, L.S., Barenco, P.V.C., Sousa, R.O., Rodrigues, R.M., Mineo, J.S., Silva, J.S. and Silva, N.M. (2013) Toxoplasma gondii soluble tachyzoite antigen triggers protective mechanisms against fatal intestinal pathology in oral infection of C57bl/6 mice. PLoS One, 8(9): e75138.

7. Fuentes-Castro, B.E., Reyes-García, J.G., Valenzuela-Vargas, M.T. and Martínez-Gómez, F. (2017) Histopathology of murine toxoplasmosis under treatment with dialyzable leukocyte extract. Mem. Inst. Oswaldo Cruz, 112(11): 741-747.

8. Maksimov, P., Buschtöns, S., Herrmann, D.C., Conraths, F.J., Görlich, K., Tenter, A.M., Dubey, J.P., Nagel-Kohl, U., Thoms, B., Bötcher, L., Kühne, M. and Schares, G. (2011) Serological survey and risk factors for Toxoplasma gondii in domestic ducks and geese in Lower Saxony, Germany. Vet. Parasitol., 182(2-4): 140-149.

9. Heider, M., Henriques-Pons, A., Tedesco, R. and Barbosa, H. (2010) Viability and infectivity analysis of Toxoplasma gondii under axenic conditions. Sci. Med., 20(1): 93-98.

10. Johno, K., Sano, R., Toda, N. and Yamada, G. (1958) The survival of Toxoplasma gondii in various suspending media. Med. Entomol. Zool., 9(2): 97.

11. Räisänen, S.A. (1978) The importance of trophozoites in transmission of toxoplasmosis: Survival and pathogenicity of Toxoplasma gondii trophozoites in liquid media. Med. Hypotheses, 4(4): 367-375.

12. El-Bahy, M.M., Khalifa, M.M. and Méabed, E.M.H. (2018) Toxoplasma gondii: Prolonged in vitro maintenance of virulent tachyzoites in fluid media at low temperatures. Alexandria J. Med., 54(4): 511-515.

13. Kalani, H., Daryani, A., Sharif, M., Ahmadpour, E., Alizadeh, A., Nasrolahei, M., Sarvi, S., Kalani, F. and Faridnia, R. (2016) Comparison of eight cell-free media for maintenance of Toxoplasma gondii tachyzoites. Iran. J. Parasitol., 11(1): 104-109.

14. Koethe, M., Schade, C., Fehlhaber, K. and Ludewig, M. (2017) Survival of Toxoplasma gondii tachyzoites in simulated gastric fluid and cow's milk. Vet. Parasitol., 233(2017): 111-114.

15. Nguyen, T.T., Kengradomkij, C. and Inpankaew, T. (2021) Detection of antibodies to Toxoplasma gondii among owned dogs in Cambodia. Food Waterborne. Parasitol., 22(2021): e00103.

16. Strober, W. (2001) Trypan blue exclusion test of cell viability. Curr. Protoc. Immunol., Appendix 3 (2001): Appendix 3B..

17. R Core Team. (2020) R: A Language and Environment for Statistical Computing: R Foundation for Statistical Computing, Vienna, Austria. Available from: https:// www.r-project.org. Retrieved on 12-06-2020.

18. Bajelan, S., Bahreini, M.S., Asgari, Q. and Mikaeili, F. (2020) Viability and infectivity of Toxoplasma gondii tachyzoites exposed to Butanedione monoxime. J. Parasit. Dis., 44(4): 1-7.

19. Blumberg, N., Cholette, J.M., Pietropaoli, A.P., Phipps, R., Spinelli, S.L., Eaton, M.P., Noronha, S.A., Seghatchian, J., Heal, J.M. and Refaai, M.A. (2018) $0.9 \% \mathrm{NaCl}$ (normal saline)-perhaps not so normal after all? Transfus. Apher. Sci., 57(1): 127-131

20. Li, H., Sun, S.R., Yap, J.Q., Chen, J.H. and Qian, Q. (2016) $0.9 \%$ saline is neither normal nor physiological. J. Zhejiang Univ. Sci. B., 17(3): 181-187.

21. Hamer, W. and $\mathrm{Wu}, \mathrm{Y}$. (1972) Osmotic coefficients and mean activity coefficients of uni-univalent electrolytes in water at $25^{\circ}$ C. J. Phys. Chem. Ref. Data, 1(4): 1047-1100.

22. Miranda, F.J., Souza, D.B., Frazão-Teixeira, E., Oliveira, F.C., Melo, J.C., Mariano, C.M., Albernaz, A.P., Carvalho, E.C., Oliveira, F.C., Souza, W.D. and DaMatta, R.A. (2015) Experimental infection with the Toxoplasma gondii ME-49 strain in the Brazilian BR-1 mini 
pig is a suitable animal model for human toxoplasmosis. Mem. Inst. Oswaldo Cruz., 110(1): 95-100.

23. Nguyen, T.D., de Kesel, M., Bigaignon, G., Hoet, P., Pazzaglia, G., Lammens, M. and Delmee, M. (1996) Detection of Toxoplasma gondii tachyzoites and bradyzoites in blood, urine, and brains of infected mice. Clin. Diagn. Lab. Immunol., 3(6): 635-639.

24. Silveira, C., Vallochi, A., Silva, U., Muccioli, C., Holland, G., Nussenblatt, R.B., Belfort, R. and Rizzo, L. (2011) Toxoplasma gondii in the peripheral blood of patients with acute and chronic toxoplasmosis. Br. J. Ophthalmol., 95(3): 396-400.

25. Unno, A., Kachi, S., Batanova, T.A., Ohno, T., Elhawary, N., Kitoh, K. and Takashima, Y. (2013) Toxoplasma gondii tachyzoite-infected peripheral blood mononuclear cells are enriched in mouse lungs and liver. Exp. Parasitol., 134(2): 160-164.

26. Wastling, J.M., Nicoll, S. and Buxton, D. (1993) Comparison of two gene amplification methods for the detection of Toxoplasma gondii in experimentally infected sheep. $J$. Med. Microbiol., 38(5): 360-365.

27. Reddi, B.A.J. (2013) Why is saline so acidic (and does it really matter?) Int. J. Med. Sci., 10(6): 747-750.

28. Pott, S., Koethe, M., Bangoura, B., Zöller, B., Daugschies, A., Straubinger, R.K., Fehlhaber, K. and Ludewig, M. (2013) Effects of $\mathrm{pH}$, sodium chloride, and curing salt on the infectivity of Toxoplasma gondii tissue cysts. J. Food. Prot., 76(6): 1056-1061.

29. Clough, B. and Frickel, E.M. (2017) The toxoplasma parasitophorous vacuole: An evolving host-parasite frontier. Trends Parasitol., 33(6): 473-488.

30. Gupta, N., Hartmann, A., Lucius, R. and Voelker, D.R. (2012) The obligate intracellular parasite Toxoplasma gondii secretes a soluble phosphatidylserine decarboxylase. $J$. Biol. Chem., 287(27): 22938-22947.

31. Sinai, A.P. (2014) The Toxoplasma gondii parasitophorous vacuole membrane: A multifunctional organelle in the infected cell. In: Weiss, L.M. and Kim, K., editors. Toxoplasma gondii. $2^{\text {nd }}$ ed., Ch. 11. Academic Press, Boston. p375-387.

32. Schwab, J.C., Beckers, C.J. and Joiner, K.A. (1994) The parasitophorous vacuole membrane surrounding intracellular Toxoplasma gondii functions as a molecular sieve. Proc. Natl. Acad. Sci. U. S. A., 91(2): 509-513.
33. Blume, M. and Seeber, F. (2018) Metabolic interactions between Toxoplasma gondii and its host. F1000Res, 7: 1719.

34. Gold, D.A., Kaplan, A.D., Lis, A., Bett, G.C., Rosowski, E.E., Cirelli, K.M., Bougdour, A., Sidik, S.M., Beck, J.R., Lourido, S., Egea, P.F., Bradley, P.J., Hakimi, M.A., Rasmusson, R.L. and Saeij, J.P. (2015) The Toxoplasma dense granule proteins GRA17 and GRA23 mediate the movement of small molecules between the host and the parasitophorous vacuole. Cell Host Microbe, 17(5): 642-652.

35. MacRae, J.I., Sheiner, L., Nahid, A., Tonkin, C., Striepen, B. and McConville, M.J. (2012) Mitochondrial metabolism of glucose and glutamine is required for intracellular growth of Toxoplasma gondii. Cell Host Microbe, 12(5): 682-692.

36. Coppens, I., Dunn, J.D., Romano, J.D., Pypaert, M., Zhang, H., Boothroyd, J.C. and Joiner, K.A. (2006) Toxoplasma gondii sequesters lysosomes from mammalian hosts in the vacuolar space. Cell, 125(2): 261-274.

37. Dou,Z., McGovern, O.L., di Cristina, M. and Carruthers, V.B. (2014) Toxoplasma gondii ingests and digests host cytosolic proteins. $m$ Bio, 5(4): e01188-e01214.

38. Diab, M.R. and El-Bahy, M.M. (2008) Toxoplasma gondii: Virulence of tachyzoites in serum free media at different temperatures. Exp. Parasitol., 118(1): 75-79.

39. Dean, P., Major, P., Nakjang, S., Hirt, R.P. and Embley, T.M. (2014) Transport proteins of parasitic protists and their role in nutrient salvage. Front. Plant. Sci., 5: 153.

40. Khan, K. and Elia, M. (1991) Factors affecting the stability of L-glutamine in solution. Clin. Nutr., 10(4): 186-192.

41. Thermofisher Scientific. (2021b) L-Glutamine and GlutaMAX Cell Culture Supplements. Available from: https://www.thermofisher.com/th/en/home/life-science/ cell-culture/mammalian-cell-culture/media-supplements/ glutamax-media.html. Retrieved on 28-06-2020.

42. Thermofisher Scientific. (2021a) Balanced Salt Solutions. Available from: https://www.thermofisher.com/th/en/home/ life-science/cell-culture/mammalian-cell-culture/reagents/ balanced-salt-solutions.html. Retrieved on 28-06-2020.

43. Gebb, C., Clark, J.M., Hirtenstein, M.D., Lindgren, G.E., Lundgren, B.J., Lindskog, U. and Vretblad, P.A. (1984) Alternative surfaces for microcarrier culture of animal cells. In: Acton, R.T. and Daniel, L.J., editors. Eukaryotic Cell Cultures: Basics and Applications. Springer, New York, US. p151-167. 